\title{
First principles study of the electronic properties of MgO under pressure
}

\author{
Cui Zhou ${ }^{1,}$, , Zhiqiang Liu ${ }^{2}$, Rui Kang ${ }^{1}$, Yue Huang ${ }^{1}$, Qingbo Wang ${ }^{3}$ \\ ${ }^{1}$ No. 3 Department, Air Force Early Warning Academy, Wuhan 430019, China \\ ${ }^{2}$ College of Science, Jiujiang University, Jiujiang 332005, China \\ ${ }^{3}$ School of Mathematics and Physics, China University of Geosciences (Wuhan), Wuhan 430074, \\ China \\ acuizhou2014@163.com
}

Keywords: MgO, Pressure, First principles, Electronic properties.

\begin{abstract}
We have studied the electronic properties of MgO by first principles (CASTEP program). The calculated band gap of $\mathrm{MgO}$ shows $\mathrm{MgO}$ is an insulator. Pressure increases the band gap from 4.253 to $7.585 \mathrm{eV}$. The band above the Fermi level shifts to higher energy, while the band below the Fermi level shifts to lower energy. The shift comes from effect of pressure. The density of states (DOS) have been calculated and the relations between band and DOS have been discussed. The DOS also have shift and the shifts is according to the shifts in band. The electronic properties have also been calculated. We also find the pressure shifts the electronic properties to higher energy. The electronic shift come from the increased band gap. Our result provides a reference for future experiments.
\end{abstract}

\section{Introduction}

$\mathrm{MgO}$ is an important mineral in the mantle [1-3]. MgO can be widely used in industry. For example, $\mathrm{MgO}$ also is an excellent insulator [4] and carrier of catalyst [5]. MgO can also be used as expansive cement and concrete [6]. As an important mineral, the properties of $\mathrm{MgO}$ under pressure is important to identify some geological process. With the development of high-pressure equipment, experts devote to studying the properties of materials under pressure [7-9]. The high-pressure equipment is relative expensive for operation and maintenance. Few laboratories in our world have these equipment. These hinder the studies about the properties of materials under pressure.

On the other hand, First principles is a powerful tool to study the properties of materials under pressure [10-12]. With the develop of first principles [13] and high performance computer, First principles method is becoming more and more feasible to study material properties under pressure. So, more and more experts use computers and first principles to study material electronic properties under pressure. In this paper, we intend to study the electronic properties of MgO under pressure.

\section{Computational details}

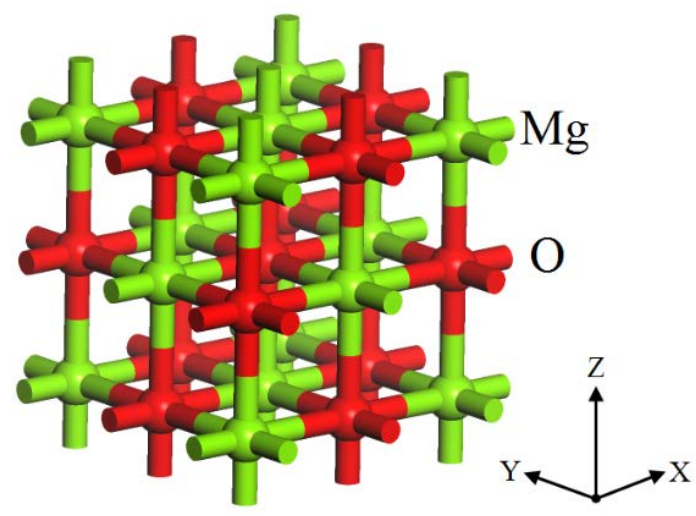

Fig. 1 The scheme crystal structure of MgO. 
Our calculations were performed by the Cambridge Sequential Total Energy Package (CASTEP) program, which is based on the Density-functional theory (DFT). The crystal of MgO showed in Fig. 1. It belongs to FM-3M space group, which was used in our calculations. The used valence electrons are $3 s^{2}$ and $2 p^{4}$. The ultrasoft pseudopotential was used in our calculations. Our calculations were carried out in a reciprocal space. After test, the used $k$-points and cut-off energy $6 \times 6 \times 6$ and $400 \mathrm{eV}$. The SCF tolerance is $1 * 10^{-5} \mathrm{eV} / \mathrm{atom}$. We chose typical pressures $(0$ and $80 \mathrm{GPa})$ and used them in our calculations. We first optimized the crystal structure and then calculated the electronic properties.

\section{Results and discussions}

Structure and band. In this section, we first discuss the structure and then discuss the band. At 0 GPa, the optimized lattice parameters are $a=b=c=4.295 \AA$ and $\alpha=\beta=\gamma=90^{\circ}$. On the other hand, the optimized lattice parameters are $a=b=c=3.884 \AA$ and $\alpha=\beta=\gamma=90^{\circ}$ at $80 \mathrm{GPa}$. From the different parameters under different pressure, the crystal angle is unchanged. The lattice constants decrease with increasing pressure and the ratio of decrease is as high as $10 \%$. The compression will induce a changes in the electronic properties. Our results are in line with the other results. The reliable constants can guarantee our calculations are reliable.

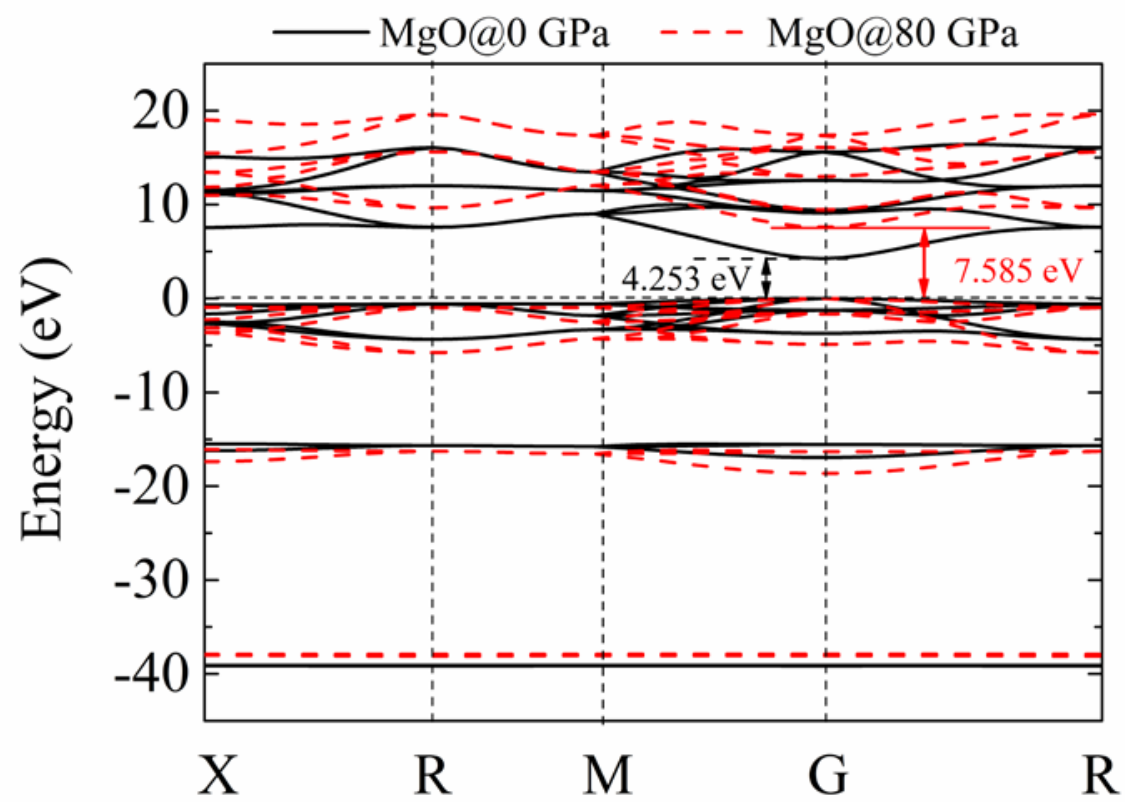

Fig. 2. The calculated band of $\mathrm{MgO}$ at 0 and $80 \mathrm{GPa}$.

Density of states. The band and density of states (DOS) relate closely to the optical properties of MgO. The band also relates closely to the DOS. In this section, we will calculate the DOS and discuss the relation between band and DOS. The relation between DOS and optical properties will be discussed in the next section. The related calculations also performed under 0 and $80 \mathrm{GPa}$. The results are shown in Fig. 3.

We first discuss the total DOS at 0 and $80 \mathrm{GPa}$ in Fig. 3a. The total DOS in Fig 3a shift to higher energy with pressure increase from 0 to $80 \mathrm{GPa}$, which is according to the shift of our calculated band. The according energy of the DOS constitute the according band. Except the shifts of the DOS, the correspondence between the band and DOS is similar. We only give the correspondence at $0 \mathrm{GPa}$ and that at $80 \mathrm{GPa}$ is similar. The DOS above the Fermi level mainly come from $\mathrm{Mg} 3 s, 3 p$ and $\mathrm{O} 2 p$, which constitute the conduction band. The total DOS (TDOS) between $-5.0 \sim 0 \mathrm{eV}$ come from $\mathrm{O} 2 p$ state, which constitutes the according valence band. The TDOS around $-15.6 \mathrm{eV}$ come from $\mathrm{O} 2 \mathrm{~s}$ state, which forms the according valence band. The TDOS at $-39.1 \mathrm{eV}$ come from $\mathrm{Mg} 2 p$ state, which constitutes the deep valence band. The correspondence is important to identify the states. 

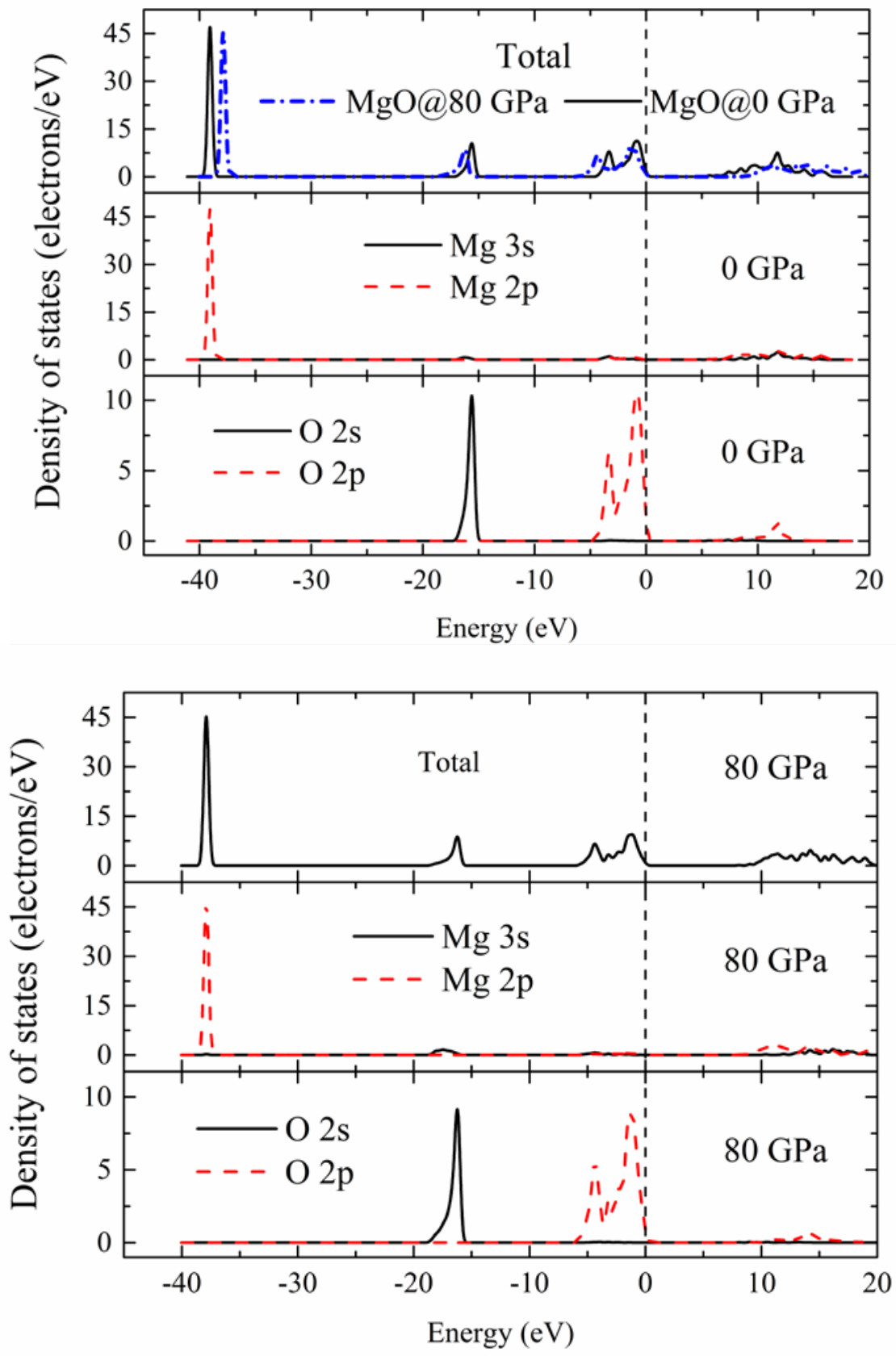

Fig. 3. The density of states (DOS) and partial DOS (PDOS) of MgO at 0 and $80 \mathrm{GPa}$.

\section{Conclusions}

In this paper, we have calculated the electronic properties of MgO under pressure (0 and $80 \mathrm{GPa}$ ). The structure has been optimized and the bands have been discussed. The band gap increases when pressure increases from 0 to $80 \mathrm{GPa}$. The band gap shows $\mathrm{MgO}$ is an insulator. The electronic density of states (DOS) have been calculated and the relation between bands and DOS has been discussed. The electronic properties has also been calculated and discussed, which can be used to identify some geological process. Our studies provide a reference and stimulate the future experiments.

\section{Acknowledgments}

The Project was supported by the National Natural Science Foundation of China (41402034). 


\section{Reference}

[1] C. Herzberg, K. Condie, J. Korenaga, Thermal history of the Earth and its petrological expression, Earth Planet. Sci. Lett. 292 (2010) 79-88.

[2] W. Tian, I.H. Campbell, C.M. Allen, P. Guan, W. Pan, M. Chen, H. Yu, W. Zhu, The Tarim picrite-basalt-rhyolite suite, a Permian flood basalt from northwest China with contrasting rhyolites produced by fractional crystallization and anatexis, Contrib. Mineral. Petrol. 160 (2010) 407-425.

[3] C. Diwu, Y. Sun, A. Guo, H. Wang, X. Liu, Crustal growth in the North China Craton at similar to $2.5 \mathrm{Ga}$ : Evidence from in situ zircon $\mathrm{U}-\mathrm{Pb}$ ages, Hf isotopes and whole-rock geochemistry of the Dengfeng complex, Gondwana Res. 20 (2011) 149-170.

[4] S. Ikeda, J. Hayakawa, Y.M. Lee, F. Matsukura, Y. Ohno, T. Hanyu, H. Ohno, Magnetic tunnel junctions for spintronic memories and beyond, Ieee Transactions on Electron Devices 54 (2007) 991-1002.

[5] G. Trunfio, F. Arena, Deactivation Pattern of a "Model" Ni/MgO Catalyst in the Pre-Reforming of n-Hexane, Catalysts 4 (2014) 196-214.

[6] L. Mo, M. Deng, M. Tang, A. Al-Tabbaa, MgO expansive cement and concrete in China: Past, present and future, Cem. Concr. Res. 57 (2014) 1-12.

[7] L. Song, L. Ci, H. Lu, P.B. Sorokin, C. Jin, J. Ni, A.G. Kvashnin, D.G. Kvashnin, J. Lou, B.I. Yakobson, P.M. Ajayan, Large Scale Growth and Characterization of Atomic Hexagonal Boron Nitride Layers, Nano Lett. 10 (2010) 3209-3215.

[8] T.-S. Wong, S.H. Kang, S.K.Y. Tang, E.J. Smythe, B.D. Hatton, A. Grinthal, J. Aizenberg, Bioinspired self-repairing slippery surfaces with pressure-stable omniphobicity, Nature 477 (2011) 443-447.

[9] S.-J. Yoon, J.W. Chung, J. Gierschner, K.S. Kim, M.-G. Choi, D. Kim, S.Y. Park, Multistimuli Two-Color Luminescence Switching via Different Slip-Stacking of Highly Fluorescent Molecular Sheets, J. Am. Chem. Soc. 132 (2010) 13675-13683.

[10] K.R. Babu, C.B. Lingam, S. Auluck, S.P. Tewari, G. Vaitheeswaran, Structural, thermodynamic and optical properties of MgF2 studied from first-principles theory, J. Solid State Chem. 184 (2011) 343-350.

[11] P.D. Borges, L.M.R. Scolfaro, H.W. Leite Alves, E.F. da Silva, Jr., DFT study of the electronic, vibrational, and optical properties of SnO2, Theor. Chem. Acc. 126 (2010) 39-44.

[12] A. Hao, X. Yang, X. Wang, Y. Zhu, X. Liu, R. Liu, First-principles investigations on electronic, elastic and optical properties of $\mathrm{XC}(\mathrm{X}=\mathrm{Si}, \mathrm{Ge}$, and $\mathrm{Sn})$ under high pressure, J. Appl. Phys. 108 (2010).

[13] S. Guindon, J.-F. Dufayard, V. Lefort, M. Anisimova, W. Hordijk, O. Gascuel, New Algorithms and Methods to Estimate Maximum-Likelihood Phylogenies: Assessing the Performance of PhyML 3.0, Syst. Biol. 59 (2010) 307-321. 